\title{
ELECTROPHYSIOLOGICAL FINDINGS IN CORTICAL BLINDNESS. REPORT OF A CASE ${ }^{1,2}$
}

\author{
K. A. KoOI, M.D. AND F. W. Sharbrough III, M.D. \\ Laboratory of Electroencephalography, Neuropsychiatric Institute and Department of Neurology, University of Michigan \\ Medical Center, Ann Arbor, Mich. (U.S.A.)
}

(Accepted for publication: October 5, 1965)

\section{INTRODUCTION}

Cortical blindness is a relatively rare condition which is characterized clinically by complete loss or gross impairment of vision, normal pupillary reactions and normal appearance of the ocular fundi. Knowledge about its cerebral electrophysiological correlates is as yet scanty (Williams and Reynell 1945; Cohn 1949; Bergman 1957). Contributing to this lack of information in man has been an inability to quantify and, indeed, even detect with any degree of consistency, cerebral responses to external visual and other types of stimuli. The introduction of response averaging has led to renewed interest in the possibility that information of diagnostic value might be provided by evoked response studies. Objective measures of central deficit may be needed when the question of cortical blindness is being entertained, because of uncertainty as to whether the patient's symptoms are "organic" or "psychogenic" in nature.

This report is directed towards describing evoked responses to light and sound in a case of cortical blindness secondary to head injury.

\section{CASE REPORT}

\section{Clinical data}

L.S., a 33 year old male, sustained multiple contusions of the head, shoulders and legs and a Colle's fracture of his right forearm in an automobile accident for which he had complete amnesia. When admitted to the local hospital, the patient was unconscious and pupils were unreactive to light. The right eye was fixed; the left eye moved slowly in a random fashion. There was ptosis of the right eyelid. Babinski signs were absent. One hour following admission, the patient's right pupil was noted to react to light, and body movement was noted for the first time. Over a 2-week period, the patient's level of consciousness gradually improved but he was completely insensitive to any type of visual stimuli.

The patient was transferred to the University Hospital 16 days following the accident. On admission, his chief complaint was "everything looks milky". Significant

\footnotetext{
${ }^{1}$ Supported in part by U.S.P.H.S. Grant NB 02560.

2 Portions of this paper were read at the meeting of the Central Association of Electroencephalographers, Iowa City, Iowa, April 2-3, 1965.
}

neurological findings were as follows. Mental status: oriented as to person, not time and place, confabulation during visual testing. Cranial nerves: no perception of light or movement, pupils reactive OU, EOM intact without nystagmus, slight right facial weakness. Motor system: intact. Sensory system: intact. Reflexes: equal and active bilaterally, Babinski signs absent, Chaddock signs present bilaterally, abortive clonus greater on right. Ocular examination in the Department of Ophthalmology was normal. Skull films: no evidence of intracranial abnormality, midline pineal. Lumbar puncture: initial pressure $170 \mathrm{~mm}$, final pressure $120 \mathrm{~mm}$; fluid clear and colorless; 4 WBC; total protein $40 \mathrm{mg} \%$; sugar $66 \mathrm{mg} \%$; Kahn serological and colloidal gold studies negative. Serum electrolytes, creatinine, BUN, bilirubin, alkaline phosphatase, BSP and prothrombin concentrations: normal.

Mentation gradually improved during the patient's hospital stay. He continued to have a mild organic brain syndrome which fluctuated in degree up to the time of his discharge. He was able to perceive light on the 15th day of his hospitalization at the University Hospital. A few days later he was able to detect movement but continued to be unable to perceive form or color to the time of discharge, approximately one month from the time of admission.

\section{Electrophysiological findings}

Technique. Resting EEGs were recorded eyes closed and eyes open, utilizing conventional electrode placements and montages.

Details of our visual evoked response procedure have been previously outlined (Kooi and Bagchi 1964). Subjects are routinely tested eyes open to stabilize level of alertness and avoid interindividual variability introduced by differences in lid thickness and vascularity. In the systematized portion of the procedure, flashes are randomized and spaced at 4-6 sec intervals, thereby avoiding additive response "carry-over" effects and permitting return of pupils to baseline size and recovery of visual system elements. The lamp encasement (Grass PS-2 photic stimulator) has been modified to eliminate awareness of the sound which normally accompanies each flash. Two accumulations of 50 responses (8 averaging channels), analysis time $250 \mathrm{msec}$, and 1 of 100 , analysis time 125 msec, provide the basic data. Consistency of response morphology is determined by repetitions of the accumulations for key electrode placements. Runs in which param- 

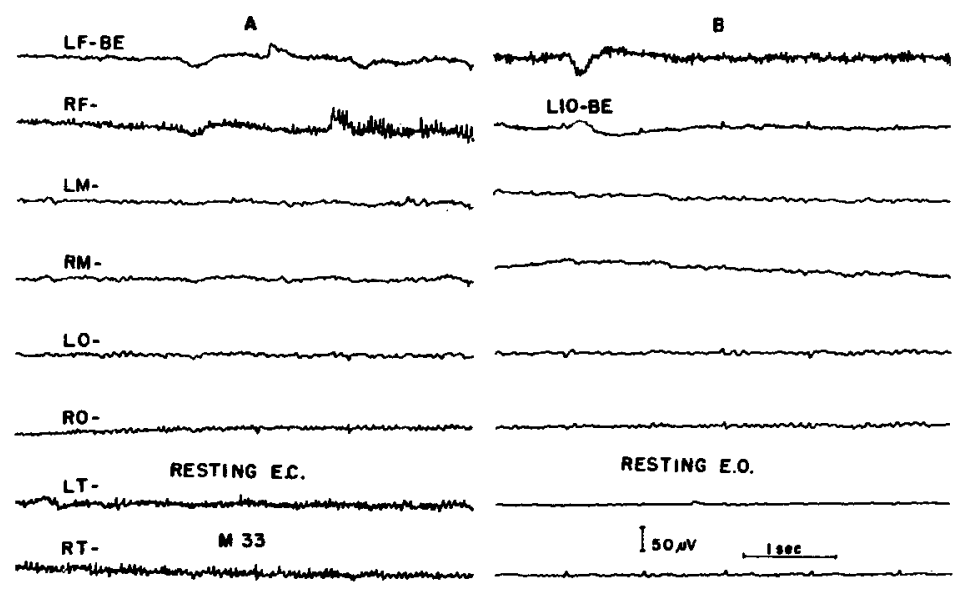

c

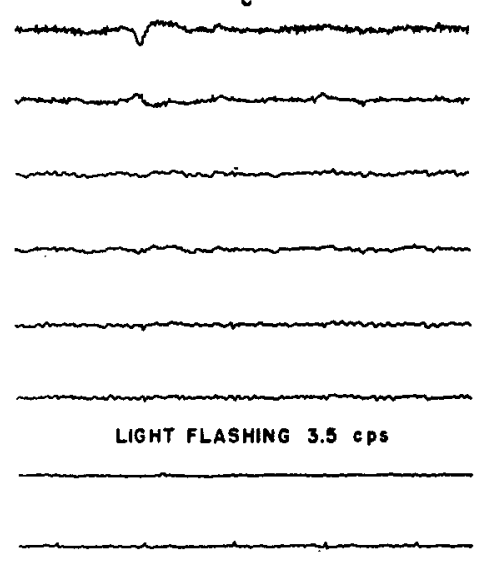

Fig. 1

$A$ : Illustration of maximal rhythmicity and amplitude of the alpha rhythm attained during 4 EEG records. $B$ and $C:$ Pattern unchanged when eyes open or by light stimulation.

In this and subsequent figures: $\mathrm{F}$, frontal; IO, infraorbital; $\mathrm{M}$, motor; $\mathrm{O}$, occipital; $\mathrm{T}$, temporal; $\mathrm{BE}$, both ears; CERV, posterior low midcervical; $L$, left; $R$, right; $n$, number of responses averaged; $d w t$, computer address dwell time; P.S., photic stimulation; E.O., eyes open; E.C., eyes closed.

A

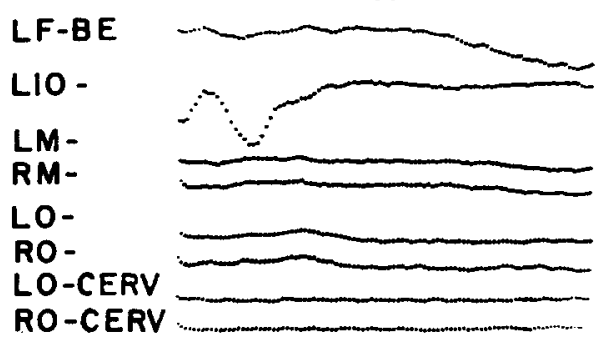

n $300 \mathrm{dw}+2.5 \mathrm{msec}$

P.S. 3.5 cps
B

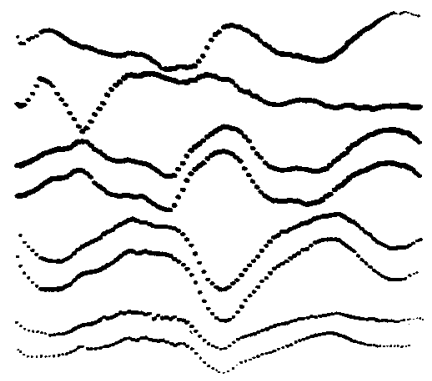

c
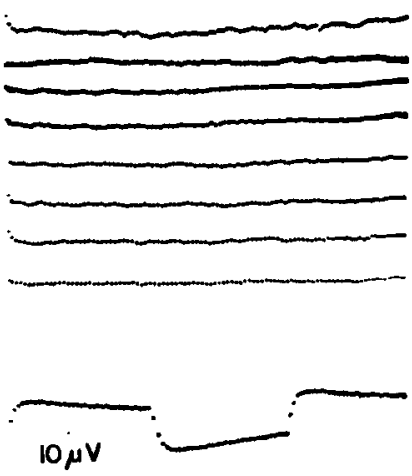

Fig. 2

Analysis period $250 \mathrm{msec}$. Light flash coincides with initiation of trace. $A$ : Response of patient. Rhythmic flashes. Face of flash lamp $5 \mathrm{~cm}$ from open eyes. Pupils symmetrical, $4 \mathrm{~mm}$ OU. ERG (LIO) well developed. LO-BE and RO-BE reveal a small amplitude surface-negative wave during the initial half of the trace, not evident in LO-CERV and RO-CERV linkages. This wave could not be definitely identified in responses obtained when the stimulus was randomized (see Fig. 3). B: Response of normal male of approximately same age with all conditions of the examination identical. $C$ : Control. Normal subject. Flash lamp in regular position and fitted with light-tight cover.

eters of stimulation and conditions of the examination are manipulated are added as indicated.

Observations. Electroencephalographic and evoked response studies were first carried out 20 days following the accident.

On the day of examination, the subject was alert and co-operative, responding promptly to questions. He had no awareness of visual stimuli. Pupils were symmetrical, $4 \mathrm{~mm}$ in diameter. Pupillary responses were active bilaterally. There was no reaction to visual threat. A consistent but slight blink reflex was present to the intense flash of the photic stimulator.

Amplitude of the resting EEG was extremely small throughout. Occipital alpha activity of $8.5-10 \mathrm{c} / \mathrm{sec}$ ranged in between 5 and $15 \mu \mathrm{V}$. Very rarely $7-8 \mathrm{c} / \mathrm{sec}$ runs were noted. Overall, alpha incidence was less than $50 \%$, the remainder of the tracing showing nonrhythmic, mixedfrequency activity of $10 \mu \mathrm{V}$ or less. Alpha incidence was 


\section{VISUAL EVOKED RESPONSES IN CORTICAL BLINDNESS}

LIGHT PERCEPTION

INFRAORBITAL

CENTRAL

OCCIPITAL
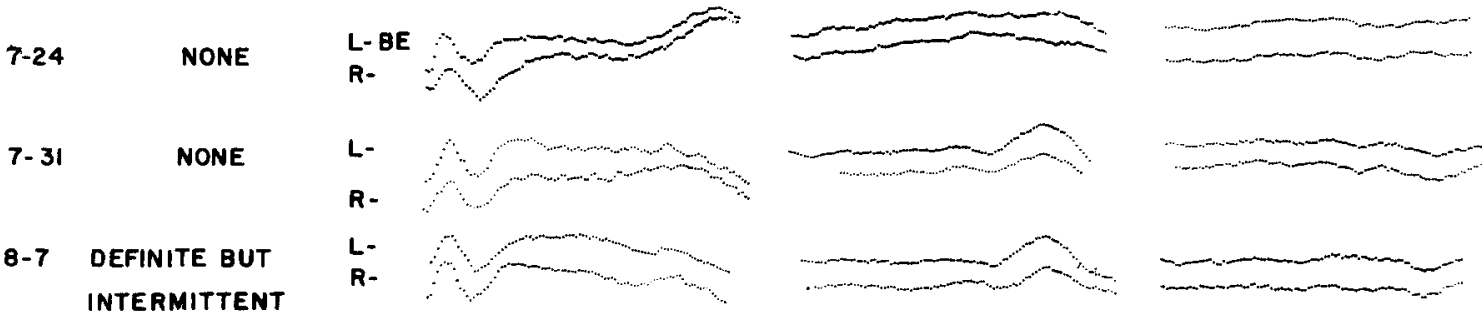

8-14

SAME

R-
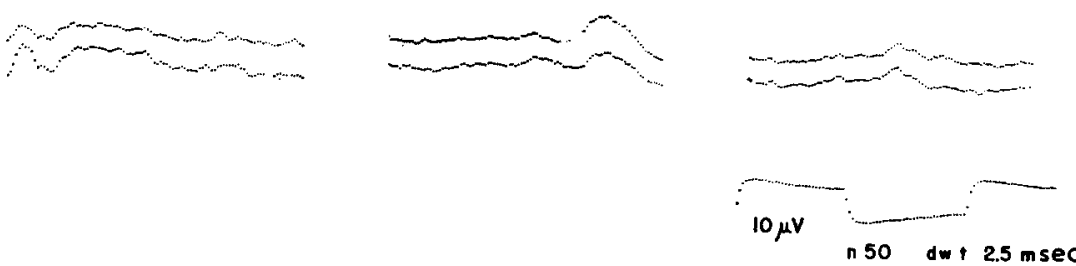

Fig. 3

Serial tracings illustrate general conformity of findings and minor evidences of improvement over a period of 4 weeks. Analysis period $250 \mathrm{msec}$. See text for further details.

not appreciably altered during periods of visual stimulation (Fig. 1). No focal signs were present and intrinsic patterns were in general symmetrical. Four series of averaged evoked responses were obtained -2 series of 50 each, 1 of 100 and 1 of 300 ( 2 series illustrated, Fig. 2 and 3). The occipital response was below $3-4 \mu \mathrm{V}$ in amplitude, none of the normal occipital deflections being recognizable. A smooth, ill-defined wave from the vertex region indicated that the afferent volley had indeed been transmitted centrally but, as in the case of the occipital response, normal features were absent. Amplitudes and latencies of both $a$ and $b$ waves of the ERG were normal.

One week later, a surface positive deflection culminating at $210-220 \mathrm{msec}$ following the flash was consistently present in the occipital region. Earlier deflections remained inconsistent. Centrally, a well formed vertex sharp wave which averaged $8-10 \mu \mathrm{V}$ with a negative peak of $195 \mathrm{msec}$ was now evident (Fig. 3). This wave was readily observed as a response to individual light flashes in the primary recording, generally several times the amplitude of central background activity which, as mentioned above, was extraordinarily small. Because of the possibility that the patient might be supersensitive to normally inaudible stimuli, special attention was directed towards eliminating sound as the effective stimulus. These studies indicated that when the flash did not fall upon the patient's eyes, even with the lamp repositioned to maximize any possible audible component of the stimulus, no central response was evident. Clinically, the patient was essentially unchanged, continuing to be unable to recognize light when tested on the ward and in the EEG Laboratory.

The patient was next studied 3 days following the first indication that vision was returning. Light perception had been definitely demonstrated although his performance was variable from time to time. Object recognition had not returned. Visual evoked responses had not changed in any major respect over those recorded a week earlier. The late occipital surface positive wave was again present, its peak latency having shortened slightly (left occipital, 1st $n 50$ trial - $200 \mathrm{msec} v s .210 \mathrm{msec}$; 2nd $n 50$ trial -195 msec vs. $220 \mathrm{msec}$ ). Vertex sharp wave peak latency to light was $192.5 \mathrm{msec}$. Further attention was directed towards evaluating the vertex response to light as opposed

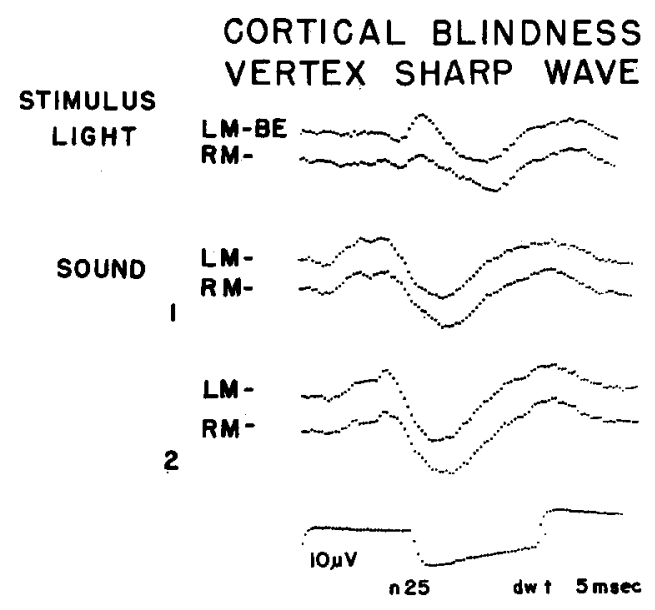

Fig. 4

Analysis period $500 \mathrm{msec}$. Vertex responses to light and sound. 1 and 2 are successive $n 25$ accumulations, all procedural variables remaining constant. Sound stimulus produced by $50 \mathrm{~V}, 0.1 \mathrm{msec}$ pulse actuating a Trimm $2 \mathrm{~K}$ impedence headset.

Electroenceph. clin. Neurophysiol., 1966, 20: 260-263 
to sound. It was found that the form of the vertex sharp wave evoked by a click was distinctly dissimilar from that to light flash, and that it culminated some $65-70 \mathrm{msec}$ earlier (Fig. 3 and 4).

During the last examination 1 week later the patient recognized the light flash approximately one-half the time. Reliability of detection was evidenced by the promptness of his responses following the flash, correct discrimination between single and two-flash stimuli, and absence of false positive responses. An occipital surface negative deflection with a peak latency of $125 \mathrm{msec}(132.5$ msec, 2nd $n 50$ trial) appeared for the first time (Fig. 3), preceding the late surface positive wave (latencies 210 msec and $200 \mathrm{msec}$ for 2 trials). The vertex sharp wave culminated at $180 \mathrm{msec}$.

\section{COMMENT}

This case establishes that an empirically normal resting EEG and the presence of occipital alpha runs are not incompatible with complete cortical blindness. The findings furnish an exception to the general rule which was deduced from a series of 12 cases studied by Bergman (1957). In all of his cases, alpha activity was "absent" when the patient was blind. Nonetheless, the low incidence and small amplitude of the alpha rhythm in our case are worthy of note, and may well indicate a substantial alpha deficit.

The outstanding clinical deficit in the patient under study was the profound visual loss, other modalities of sensation being intact. While there was some evidence of minimal recovery during 4 weeks of hospitalization, even at the time of discharge he had no useful vision, only gross changes in light intensity and movement being perceived. Suggestions of improvement in visual evoked responses were also noted, and it is tempting to speculate about a parallel course of clinical and laboratory findings. From the electrophysiological standpoint, however, even at the time of the final examination, brain responses did not remotely resemble those of individuals with normal vision. Reference is made to previously published histograms that provide a basis for comparison of a given response with probabilistic normative data (Kooi and Bagchi 1964).
An intriguing aspect of the findings was the presence of a well formed response from the vertex region in the absence of light perception and appreciable occipital response. Its preservation in this case is consistent with the widely held hypothesis that non-specific diencephalic or mesencephalic systems play a fundamental role in its genesis. The evidence suggests additionally that the occipital cortex is not an essential way station for its elaboration. That its normal morphology is partially determined by a cortico-cortical or cortico-subcortical volley cannot be entirely excluded, however, in view of its distinctly abnormal peak latency of 180-220 msec.

\section{SUMMARY}

Spontaneous cerebral activity and visual evoked responses have been described in a patient with posttraumatic cortical blindness who was studied over a period of 4 weeks. The resting occipital pattern was extremely small in amplitude but composed of frequencies largely within the normal alpha range. No evidence of light-induced alpha blocking could be detected. Averaged evoked responses derived from leads placed over the occipital poles were abnormal, in that they were rudimentary and inconsistent, none of the normal initial 5 waves being identifiable with certainty. The presence of a lightevoked, prominent vertex wave, dissimilar from that evoked by sound, was noteworthy in view of the virtual absence of an occipital response and severity of visual deficit.

\section{REFERENCES}

Bergman, P. S. Cerebral blindness. Arch. Neurol. Psychiat. (Chic.), 1957, 78: 568-584.

ConN, R. Clinical electroencephalography. McGraw-Hill, New York, 1949, 639 p.

Koor, K. A. and BAGCHI, B. K. Visual evoked responses in man: normative data. Ann. N. Y. Acad. Sci., 1964, 112: 254-269.

Williams, D. and ReYNell, J. Abnormal suppression of cortical frequencies. Brain, 1945, 68: 123-161. 